Check for updates

Cite this: RSC Adv., 2019, 9, 23390

\title{
Introducing hydrophilic ultra-thin ZIF-L into mixed matrix membranes for $\mathrm{CO}_{2} / \mathrm{CH}_{4}$ separation
}

\author{
Weifang Zhu, Xueqin Li, Yanyong Sun, Ruili Guo (D) * and Siyuan Ding \\ Mixed matrix membranes (MMMs) were developed by mixing hydrophilically modified two-dimensional \\ (2D) imidazole framework (named as hZIF-L) flakes into a Pebax MH 1657 (Pebax) matrix, and designed \\ to separate carbon dioxide/methane $\left(\mathrm{CO}_{2} / \mathrm{CH}_{4}\right)$ mixtures. The hZIF-L flakes were important for \\ increasing the effectiveness of the MMMs. First, the tannic acid (TA) etched hZIF-L flakes have a large \\ number of microporous $(1.8 \mathrm{~nm})$ and two-dimensional anisotropic transport channels, which offered \\ convenient gas transport channels and improved the permeability of $\mathrm{CO}_{2}$. Second, the TA molecules \\ provide the surface of the ZIF-L flakes with more hydrophilic functional groups such as carbonyl groups \\ $(\mathrm{C}=\mathrm{O})$ and hydroxyl groups $(-\mathrm{OH})$, which could effectively prevent non-selective interfacial voids and \\ filler agglomeration in the Pebax matrix, and also presented strong binding ability to water and $\mathrm{CO}_{2}$ \\ molecules. The satisfactory interface compatibility and affinity with the $\mathrm{CO}_{2}$ molecule promoted its \\ permeability, solubility, and selectivity. As a result, the MMMs exhibited the highest performance of gas \\ separation with the hZIF-L flake weight content of $5 \%$, at which the $\mathrm{CO}_{2}$ permeability and $\mathrm{CO}_{2} / \mathrm{CH}_{4}$ \\ selectivity were 502.44 barrer and 33.82 at $0.2 \mathrm{MPa}$ and $25^{\circ} \mathrm{C}$, respectively.
}

Received 1st June 2019

Accepted 18th July 2019

DOI: $10.1039 / c 9 r a 04147 h$

rsc.li/rsc-advances

\section{Introduction}

Gas membrane separation technology has been acknowledged as a suitable method for $\mathrm{CO}_{2}$ separation because of its low cost, small footprint requirement and simple operation. ${ }^{1}$ However, there is an inverse relationship between the permeability and selectivity of a polymer-based membrane, where an increase in permeability is accompanied by a reduction in selectivity. ${ }^{2-4}$ There are ways to improve the efficiency of the separations of polymer based membranes to overcome this trade-off effect. The current methods are new polymer design, ${ }^{5,6}$ polymer blending ${ }^{7}$ and particle filling. ${ }^{8-10}$ Among them, the introduction of inorganic fillers into polymers to generate MMMs has attracted significant interest from researchers, as the method is simple and effective.

The choice of inorganic fillers is an important factor in determining the separation performance of MMMs. A large amount of research has been devoted to the development and preparation of new inorganic fillers. Among them, the incorporation of two-dimensional (2D) materials ${ }^{11-22}$ into a polymer matrix to obtain MMMs has been the focus of research recently. Huang et al. ${ }^{23}$ prepared Pebax/GO-0.05 MMMs and the $\mathrm{CO}_{2}$ permeability and $\mathrm{CO}_{2} / \mathrm{N}_{2}$ selectivity were 113 barrer and 72 , respectively. Compared to pure Pebax membrane, the $\mathrm{CO}_{2}$

School of Chemistry and Chemical Engineering, Key Laboratory for Green Process of Chemical Engineering of Xinjiang Bingtuan, Shihezi University, Xinjiang, Shihezi 832003, China. E-mail: grli@shzu.edu.cn; Fax: +86 993 2057210; Tel: +86 993 2057277 permeability and $\mathrm{CO}_{2} / \mathrm{N}_{2}$ selectivity increased by $22.8 \%$ and $71.4 \%$, respectively. Zhao et al. ${ }^{24}$ prepared Pebax/GO MMMs and the permeability of $\mathrm{CO}_{2}$ gas was reduced by $70 \%$ compared to pure Pebax membrane at a GO content of $3.85 \mathrm{vol} \%$. Liu et al. ${ }^{19}$ prepared a Pebax-MoS $\mathrm{MMM}_{2}$ for $\mathrm{CO}_{2} / \mathrm{N}_{2}$ separation by incorporating molybdenum disulfide $\left(\mathrm{MoS}_{2}\right)$ nanosheets into the Pebax matrix. The experimental results showed that the MMM with $4.67 \mathrm{wt} \% \mathrm{MoS}_{2}$ showed good separation performance. Compared to the pure Pebax membrane, the $\mathrm{CO}_{2}$ permeability and $\mathrm{CO}_{2} / \mathrm{N}_{2}$ selectivity increased by $46.26 \%$ and $101.62 \%$, respectively. It could be concluded from the above research results that the high aspect ratio of the $2 \mathrm{D}$ materials formed highly tortuous diffusion paths for gas molecules in the polymer matrix, which increased the diffusion rate difference between large molecules and small molecules and finally improved the selectivity of the MMMs for gas mixtures. However, the permeability of the MMMs did not improve significantly. Recently, zeolitic imidazolate framework-L (ZIF-L), a new type of 2D porous MOF material, has gained extensive attention because of its specific pore size $(0.34 \mathrm{~nm})$ and strong $\mathrm{CO}_{2}$ adsorption capacity. ${ }^{25}$ The $2 \mathrm{D}$ network structure of ZIF-L was further stabilized by the interaction of the Hmim 'terminal' ligand (TL) with Hmim 'free' ligand (FL), but this van der Waals force interaction was weak. ${ }^{26,27}$ After sonication, ZIF-L was easily stripped into ultra-thin flakes with a high aspect ratio > 300. In addition, ZIF-L has a $9.4 \AA \times 7.0 \AA \times 5.3 \AA$ cushionshaped cavity between the layers, which is suitable for accommodating $\mathrm{CO}_{2}$ molecules. ${ }^{25}$ Meanwhile, the imidazole group on ZIF-L flakes can form a strong interaction with the $\mathrm{CO}_{2}$ 
molecule. ${ }^{28}$ In summary, we believed that ZIF-L was suitable for gas separation. However, there was a problem that ZIF-L's hydrophobicity and poor compatibility with the Pebax matrix needed to be improved.

TA has a strong bonding ability with metal ions and is often used as a hydrophilic surface modifier for a few materials and as an etchant for MOFs. ${ }^{29-31} \mathrm{Hu}$ et al. ${ }^{29}$ developed a simple strategy and found that phenolic acid was a unique etchant. It was possible to create voids inside the MOF while performing surface modification. They etched ZIF-8, MIL-68, and ZIF-67 with phenolic acid (i.e., gallic acid (GA) or TA). The results showed that these materials not only retained the original morphological structure but also formed a mesoporous structure after etching. Therefore, the surface functionalized auxiliary etching method could be applied to hydrophilic surface modification and making changes in the structure of ZIF-L flakes.

In this study, the functionalized auxiliary etching of ZIF-L flakes by TA was performed, and it is believed that using TA as an etchant will increase the pore size of the ZIF-L flakes. In addition, the functional modification of the surface of the ZIF-L flakes could effectively improve the issue of its hydrophobicity. The synthetic ZIF-L filler, as well as the functional modification of ZIF-L flakes by TA, and the MMMs were characterized by TEM, XRD, FT-IR spectroscopy and other analytical techniques. The effects of different fillers, contents, feed pressure and operating temperature on the performance of gas separation of the MMMs were determined.

\section{Experimental}

\subsection{Materials}

98\% zinc nitrate hexahydrate $\left(\mathrm{H}_{12} \mathrm{~N}_{20} \mathrm{O}_{12} \mathrm{Zn}\right)$ was obtained from Alfa Aesar Chemical Co., Ltd (Shanghai, China). 2-Methylimidazole (Hmim) was obtained from Adamas Reagent Co., Ltd (Shanghai, China). Ethyl alcohol was obtained from Tianjin Fuyu Fine Chemical Co., Ltd (Tianjin, China). Pebax 1657 was obtained from Shanghai Rongtian Chemical Co., Ltd (Shanghai, China). Deionized water (DI) was prepared in our laboratory and used in every case. The reagents were used as received.

\subsection{Synthesis of hZIF-L flakes}

The ZIF-L flakes were prepared by previously reported methods. ${ }^{25}$ Specifically, an aqueous zinc nitrate solution $(0.59 \mathrm{~g}$, $40 \mathrm{~mL}$ ) was added to an aqueous solution of Hmim (1.30 g, 40 $\mathrm{mL}$ ) with stirring. After $4 \mathrm{~h}$ of stirring at room temperature, the product was concentrated by centrifuging several times, washed thrice with water, and dried at $70{ }^{\circ} \mathrm{C}$ overnight in an oven. The obtained ZIF-L flakes were added to a TA solution $\left(3 \mathrm{mg} \mathrm{mL}{ }^{-1}\right)$ and stirred for $5 \mathrm{~min}$, and then allowed to stand for $7 \mathrm{~min}$. The modified ZIF-L flakes were collected by centrifugation and washed 3 times with water. As the final step, the collected product was freeze dried and kept in a desiccator. The modified ZIF-L flakes are referred to as hZIF-L flakes.

\subsection{Membrane fabrication}

All membranes were obtained by a solution-casting method (Fig. 1). ${ }^{32-34}$ The Pebax/ZIF-L- $X$ and Pebax/hZIF-L- $X$ membranes were prepared with different loadings $(1,3,5,7$ and 9 wt $\%)$ of ZIF-L flakes and hZIF-L flakes, respectively. First, a specific amount of filler was dispersed using sonication into a small amount of ethyl alcohol solution to obtain a uniform suspension. At the same time, a given mass of Pebax 1657 was dissolved in a solution mixture (ethyl alcohol to water ratio of $70 \mathrm{wt} \%$ to $30 \mathrm{wt} \%$ ) under reflux stirring for $2 \mathrm{~h}$ to obtain a polymer solution. Subsequently, the solution with uniform filler was added to the Pebax 1657 solution at $25^{\circ} \mathrm{C}$ and stirred for $3 \mathrm{~h}$. The final mixtures with different loadings of filler were cast on an ultra-flat glass plate and then dried at $25^{\circ} \mathrm{C}$ for $24 \mathrm{~h}$. To eliminate the remaining solvent, the plates were vacuumdried at $40{ }^{\circ} \mathrm{C}$ for $24 \mathrm{~h}$. Pure Pebax membrane was prepared in the same manner.

\subsection{Characterization of filler and membrane}

The morphologies of the ZIF-L flakes, pure Pebax membrane, and Pebax/hZIF-L membranes were determined by using scanning electron microscopy (SEM, SU8010). The leaf-like shape of ZIF-L flakes was observed using atomic force microscopy (AFM, Bruker). The structures of the ZIF-L flakes and hZIF-L flakes were also observed using transmission electron microscopy (TEM) on a Tecnai G2 F20 S-TWIN. The chemical structures of the ZIF-L flakes, hZIF-L flakes and membranes were characterized using Fourier-transform infrared (FT-IR) spectroscopy on a Nicolet AVATAR 360 in the range between $400 \mathrm{~cm}^{-1}$ and $4000 \mathrm{~cm}^{-1}$. The surface area and pore characteristics of the hZIF-L flakes were calculated based on the standard adsorption parameters using the BarrettJoyner-Halenda (BJH) algorithm and the Brunauer-EmmettTeller theory (BET, ASAP $2020 \mathrm{C}$ ). The crystalline properties of the ZIF-L flakes, hZIF-L flakes and membranes were measured by using X-ray diffraction (XRD, Bruker D8). The registered $2 \theta$ interval was $10^{\circ}$ to $90^{\circ}$ using $\mathrm{CuK} \alpha$ as the source of radiation. The glass transition temperature $\left(T_{\mathrm{g}}\right)$ was determined by using differential scanning calorimetry (DSC, 200, F3) from $-60{ }^{\circ} \mathrm{C}$ to $250{ }^{\circ} \mathrm{C}$ with a scanning rate of $10{ }^{\circ} \mathrm{C} \mathrm{min}^{-1}$ in a $\mathrm{N}_{2}$ atmosphere. The thermal stability of the ZIF-L flakes, hZIF-L flakes, and the membranes was examined by using thermogravimetric analysis (TGA, STA449F3) under a $\mathrm{N}_{2}$ atmosphere, using the range of $0-800{ }^{\circ} \mathrm{C}$, with a heating rate of $10^{\circ} \mathrm{C} \mathrm{min}{ }^{-1}$. The optical contact angle was determined with the sessile drop method using a Krüss Easy Drop Goniometer.

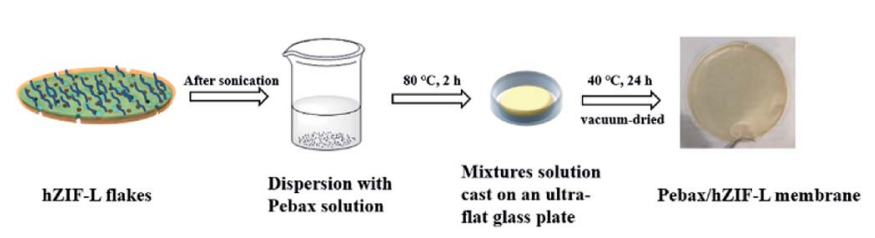

Fig. 1 Schematic representation of the synthesis of Pebax/hZIF-L membranes. 


\subsection{Gas permeation tests}

Gas permeation tests were performed under dry or humid conditions using a (constant pressure)/(variable volume method) at $25^{\circ} \mathrm{C} .{ }^{35,36}$ In the humidified permeation experiment, the sweep gas was pure hydrogen $\left(\mathrm{H}_{2}\right)$ and the feed gas was the mixed-gas. The mixed-gas was saturated with water vapor at $40{ }^{\circ} \mathrm{C}$ and then the excess moisture was condensed at room temperature in a dehumidifier. Then, the mixed-gas was driven into the membrane cell. Also, $\mathrm{H}_{2}$ was humidified by being transited through a humidifier bottle at $25{ }^{\circ} \mathrm{C}$. The composition of gases after permeation was measured using gas chromatography (GC, Agilent Technologies 7820A GC System).

The gas permeability $\left(P_{\mathrm{i}}\right.$, barrer, and 1 barrer $=10^{-10} \mathrm{~cm}^{3}$ (STP) $\mathrm{cm} \mathrm{cm}^{-2} \mathrm{~s}^{-1} \mathrm{cmHg}^{-1}$ ) could be obtained by the following eqn (1):

$$
P_{\mathrm{i}}=\frac{Q_{\mathrm{i}} l}{\Delta p_{\mathrm{i}} A}
$$

where $Q_{\mathrm{i}}$ is the volumetric flow rate $\left(\mathrm{cm}^{3} \mathrm{~s}^{-1}\right)$ (STP) of gas ' $\mathrm{i}$ ', $\Delta p_{\mathrm{i}}$ is the pressure difference of gas ' $\mathrm{i}$ ' passing through the membrane ( $\mathrm{cmHg}), l$ is the thickness of the membrane $(\mathrm{cm})$, and $A$ is the effective membrane area $\left(12.9 \mathrm{~cm}^{2}\right)$.

The mixed-gas separation factor $\left(\alpha_{\mathrm{ij}}\right)$ was estimated by (2):

$$
\alpha_{\mathrm{ij}}=\frac{P_{\mathrm{i}}}{P_{\mathrm{j}}}
$$

\section{Results and discussion}

\subsection{Characterization of hZIF-L flakes}

Fig. 2 shows that the ZIF-L flakes exhibited leaf-like shapes with sizes of about $5.9 \mu \mathrm{m} \times 2.4 \mu \mathrm{m}$ and thicknesses of about $100 \mathrm{~nm}$. After ultrasonication, the thickness of ZIF-L and hZIF-L flakes decreased to about $18 \mathrm{~nm}$ and $20 \mathrm{~nm}$, respectively, but still kept the same leaf-like morphology (Fig. 3 and 4). Hence, the ultrathin layer of ZIF-L and hZIF-L flakes was used in the follow-up experiment.

The sizes and morphologies of ZIF-L and hZIF-L flakes were characterized by using TEM, as shown in Fig. 5. A smooth

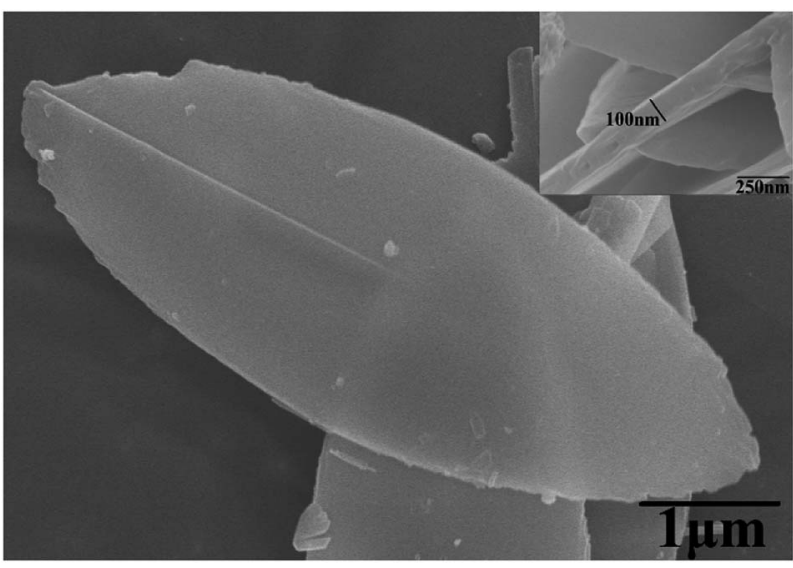

Fig. 2 SEM image of ZIF-L flakes.
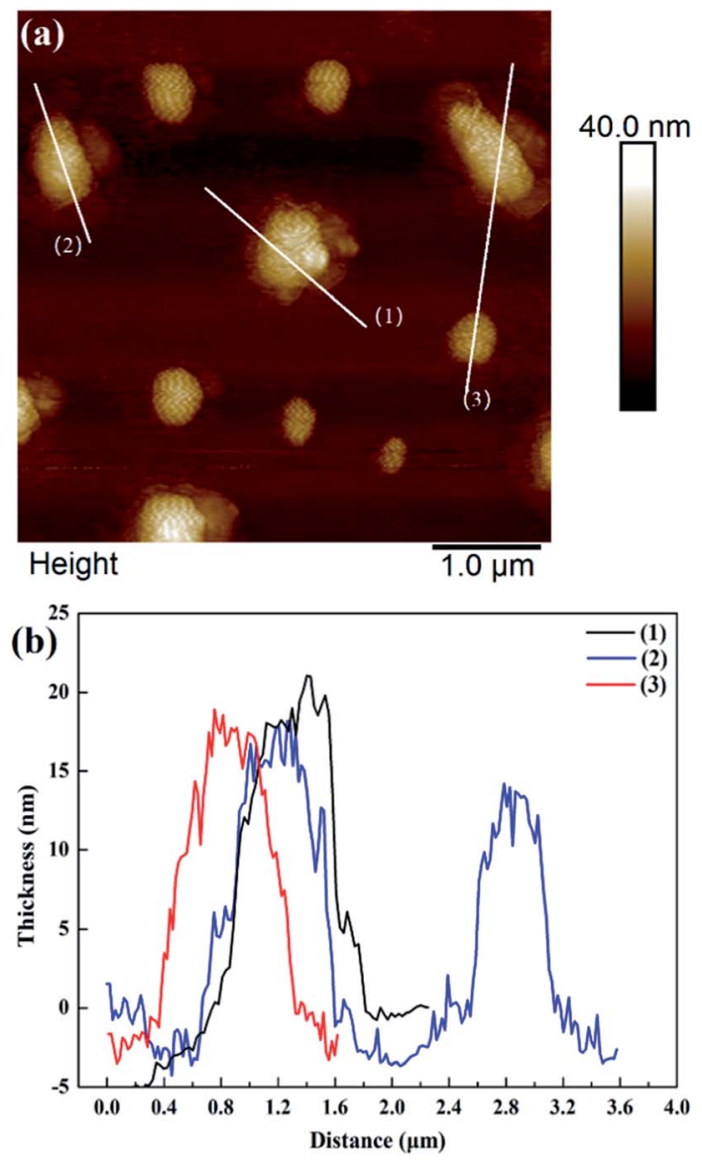

Fig. 3 (a) AFM image of ZIF-L flakes. (b) Thickness results along each white line in (a).

surface of the ZIF-L flakes could be seen, which agrees with the previous reports. ${ }^{25,37,38}$ The hZIF-L flakes retained the leaf-like morphology, but the surface of the hZIF-L flakes became rough as the TA etched the ZIF-L.

Fig. 6(a) shows that the adsorption isotherms of hZIF-L flakes belong to type I. The pore distribution shown in Fig. 6(b) indicated that fewer mesopores and many microporous structures exist in the hZIF-L flakes, with sizes of $5.6 \mathrm{~nm}$ and $1.8 \mathrm{~nm}$, respectively. The surface and pore properties of the hZIF-L flakes were as follows: a specific surface area of $87.98 \mathrm{~m}^{2}$ $\mathrm{g}^{-1}$; a pore diameter of $3.28 \mathrm{~nm}$; and a pore volume of $0.07 \mathrm{~cm}^{3}$ $\mathrm{g}^{-1}$. The pore size of ZIF-L flakes reported in the literature was $0.34 \mathrm{~nm}^{28}$

The FT-IR spectra of the ZIF-L flakes and hZIF-L flakes are shown in Fig. 7(a). Peaks were assigned as follows: $1570 \mathrm{~cm}^{-1}$, $\mathrm{C}=\mathrm{N}$ stretching; $755 \mathrm{~cm}^{-1}, \mathrm{Zn}-\mathrm{O}$ imidazole ring bending; $674 \mathrm{~cm}^{-1}, \mathrm{Zn}-\mathrm{N}$ imidazole ring bending. In the TA spectrum, peaks appeared at $1718 \mathrm{~cm}^{-1}(\mathrm{C}=\mathrm{O}$ stretching $)$ and $3412 \mathrm{~cm}^{-1}$ (-OH stretching). The characteristic peaks of ZIF-L flakes and TA also appeared in the spectrum of hZIF-L flakes. Compared to the pure TA, the peaks of hZIF-L flakes at $3412 \mathrm{~cm}^{-1}$ and $1718 \mathrm{~cm}^{-1}$ shifted to lower wavenumbers, i.e., $3366 \mathrm{~cm}^{-1}$ and $1701 \mathrm{~cm}^{-1}$, respectively. These shifts may have been caused by the chelation of the carbonyl $(\mathrm{C}=\mathrm{O})$ groups of $\mathrm{TA}$ with $\mathrm{Zn}^{2+}$ on 

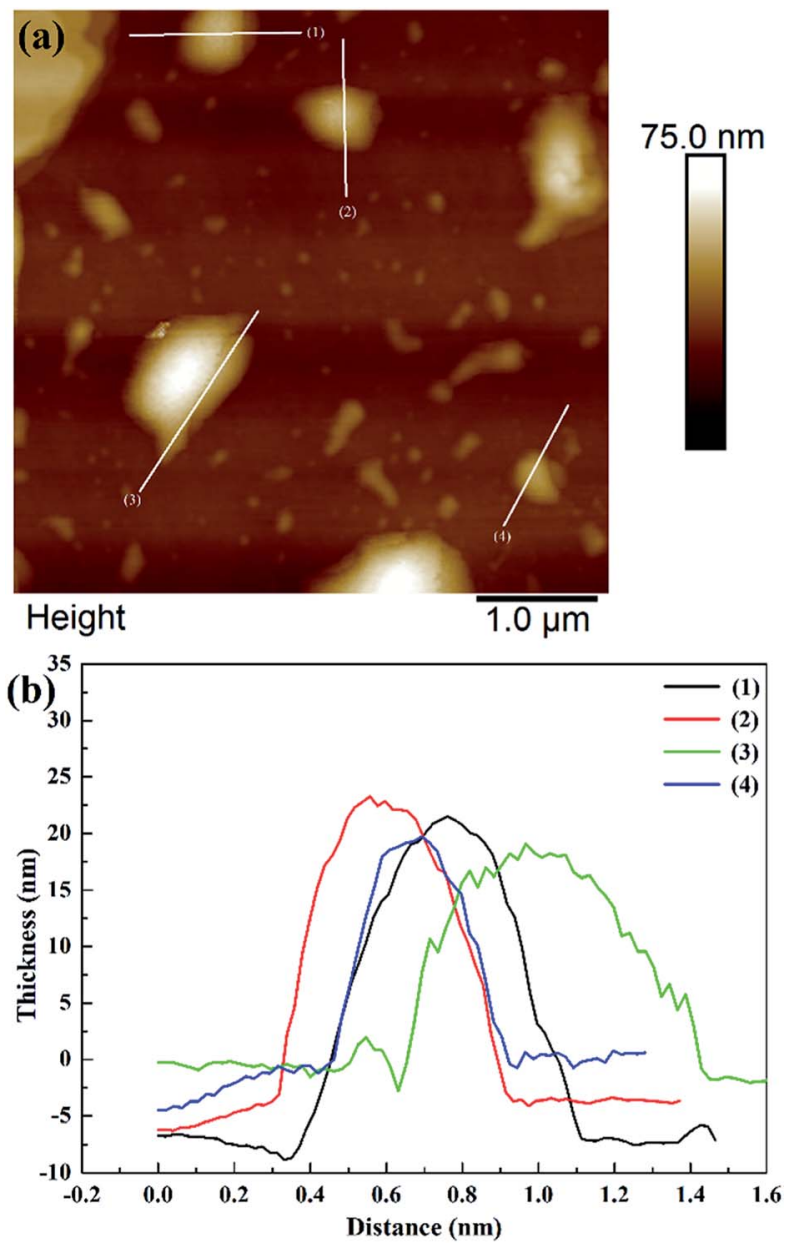

Fig. 4 AFM image of hZIF-L flakes. (a) AFM image of hZIF-L flakes. (b) Thickness results around each white line.

the surface of the ZIF-L flakes. The polar groups on the surface of the hZIF-L flakes promoted compatibility with the polymer matrix, which also enhanced the solubility of $\mathrm{CO}_{2}$ molecules.

The XRD patterns of ZIF-L and hZIF-L flakes are shown in Fig. 7(b). It could be seen from the XRD pattern that the characteristic peaks of ZIF-L and hZIF-L flakes were consistent with the characteristic peaks of the simulated ZIF-L flakes, ${ }^{39,40}$ and the patterns also confirmed the formation of ZIF-L and hZIF-L flakes with high crystallinity.
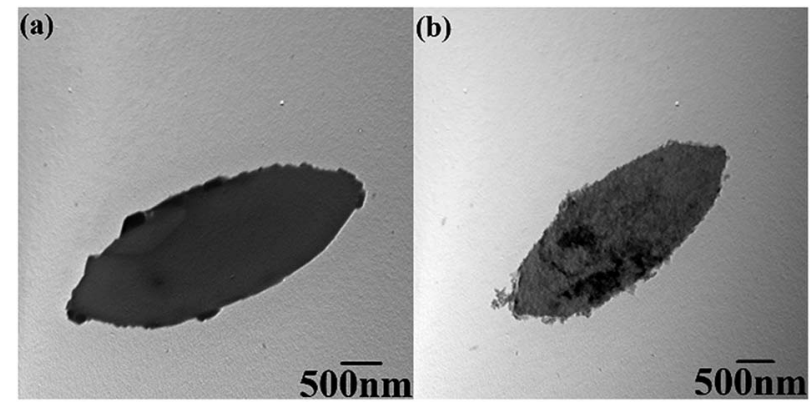

Fig. 5 TEM images of (a) ZIF-L flakes and (b) hZIF-L. flakes.
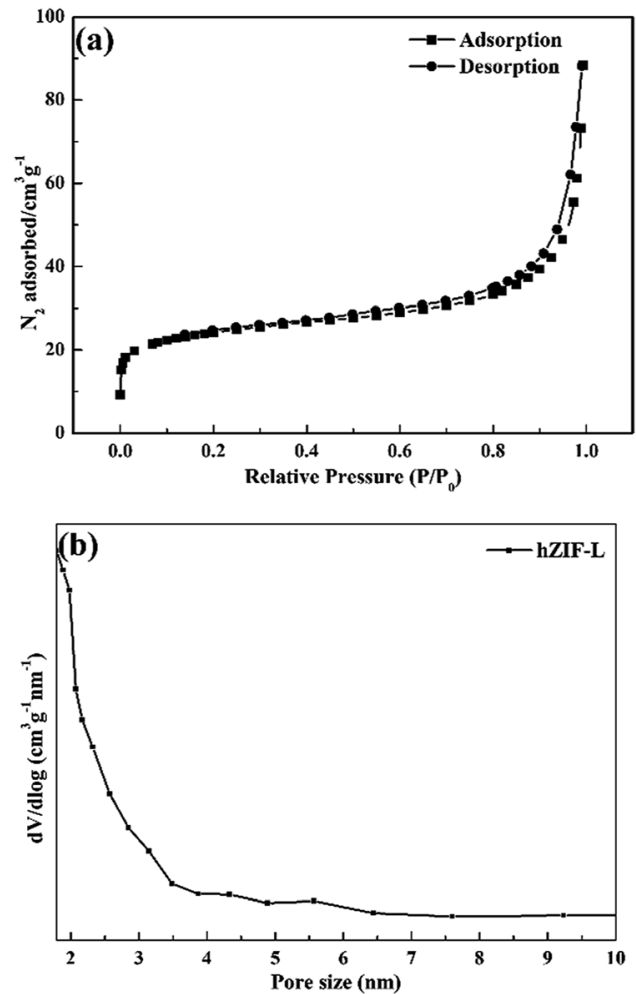

Fig. 6 (a) $\mathrm{N}_{2}$ adsorption-desorption isotherm and (b) pore size distribution of hZIF-L flakes.
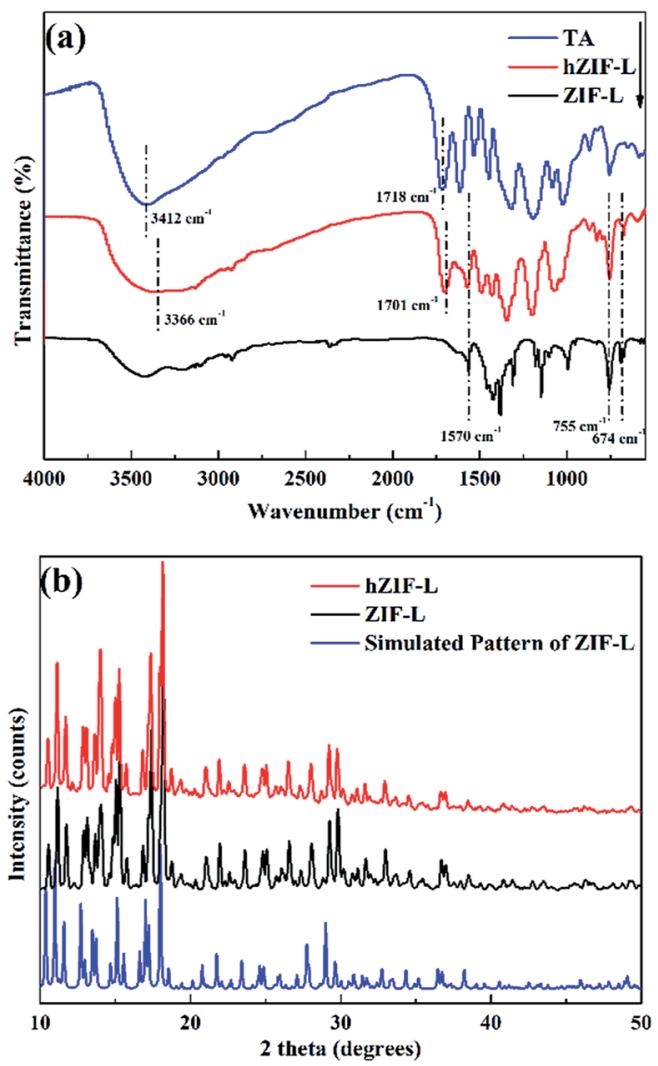

Fig. 7 (a) FT-IR spectra and (b) XRD patterns of ZIF-L and hZIF-L flakes. 
Thermogravimetric analyses of ZIF-L and hZIF-L flakes were undertaken, and the results are shown in Fig. 8. The ZIF-L and hZIF-L flakes exhibited about a $3 \mathrm{wt} \%$ weight loss before $270{ }^{\circ} \mathrm{C}$, which indicates the removal of residual solvent from the flakes. The sharp weight loss occurring between 200 and $450{ }^{\circ} \mathrm{C}$ indicates the removal of weakly linked Hmim in the hZIF-L flakes. The weight loss (24.05 wt\%) of hZIF-L flakes was much higher than that of ZIF-L flakes, which may be caused by the degradation of TA molecules on the surface of hZIF-L flakes.

\subsection{Characterization of Pebax/hZIF-L membranes}

SEM was used to study the cross-section of the pure Pebax membrane. It was observed to be even and uniform (Fig. 9). In contrast, the cross section of the Pebax/hZIF-L membranes presents a uniform horizontal strip. The hZIF-L flakes form a homogeneous dispersion in the Pebax matrix when the loading is below $5 \mathrm{wt} \%$. This phenomenon indicates that a high aspect ratio of hZIF-L flakes could be easily arranged horizontally in the Pebax matrix, which showed good compatibility with Pebax. However, when the content of filler exceeded $5 \mathrm{wt} \%$, the hZIF-L flakes showed an apparent agglomeration and were no longer present in a parallel arrangement.

The orientation of hZIF-L flakes in the Pebax matrix and the intervention effect of hZIF-L flakes on the Pebax chain crystallization region were investigated by using XRD. In Fig. 10, a broad and strong peak at $2 \theta=24.1^{\circ}$ can be observed, which may originate from the crystalline structures of the polyamide (PA6) segment of the Pebax matrix. The Pebax/hZIF-L membranes showed a lower d-spacing than that of the pure Pebax membrane, which indicates that the introduction of hZIF-L flakes limited the mobility of the Pebax chain and could improve the selectivity for gas mixtures.

The chemical structures of the pure Pebax membranes and MMMs were characterized by using FT-IR spectroscopy. As can be seen in Fig. 11, the MMMs have the same characteristic peak as the pure Pebax membrane except for the broad peak at $3512 \mathrm{~cm}^{-1}$. This peak was assigned to the $-\mathrm{OH}$ stretching vibration of the hZIF-L flakes, which indicated that the

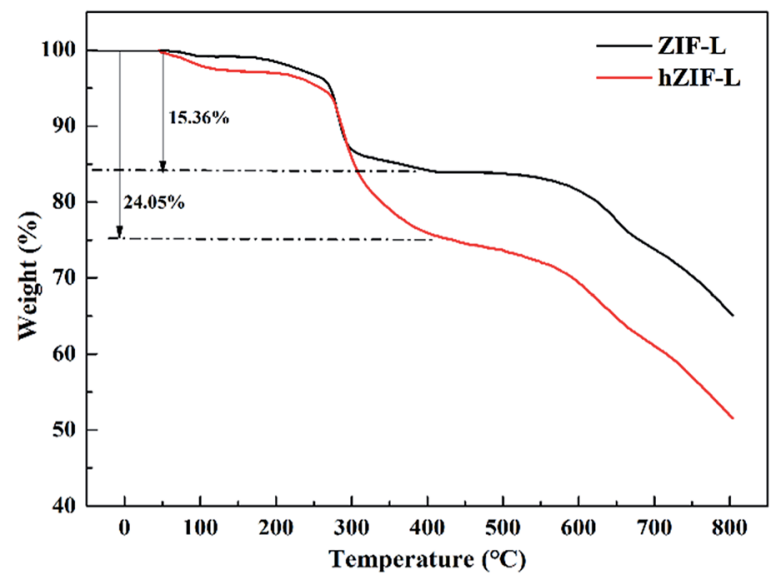

Fig. 8 TGA curves of ZIF-L and hZIF-L flakes. hydrophilic groups of TA were successfully introduced into the MMMs.

Changes in the glass-transition temperature $\left(T_{\mathrm{g}}\right)$ are often used for estimating the interfacial interaction of polymer-filler in MMMs. An increase in $T_{\mathrm{g}}$ indicates good interfacial interaction between the polymer matrix and filler and also suggests that the polymer chains have restricted mobility. The rigidification of polymer chains is conducive to an increase in selectivity. ${ }^{41}$ DSC was used to find the $T_{\mathrm{g}}$ of the Pebax and Pebax/ hZIF-L membranes. The curves are shown in Fig. 12. The Pebax and Pebax/hZIF-L membranes present similar endothermic peaks at 18.7 and $206.3^{\circ} \mathrm{C}$, which correspond to the melting of the poly[ethylene oxide] (PEO) block and PA6 block in the Pebax matrix, respectively. Fig. 12(b) shows that the Pebax/ hZIF-L membranes all have higher $T_{\mathrm{g}}$ values than the pure Pebax membrane, which indicates good interface compatibility between Pebax polymer and hZIF-L flakes. For filler contents higher than $5 \mathrm{wt} \%$, the $T_{\mathrm{g}}$ of the MMMs showed a downward trend due to the agglomeration of hZIF-L flakes in the membrane matrix. ${ }^{42}$ These results agree with the SEM observations of the MMMs (Fig. 9).

The thermal stability results of the pure Pebax and Pebax/ hZIF-L membranes with different contents of filler are shown in Fig. 13. They indicated that the membranes lose weight in a two-stage process. The first stage was due to the evaporation of the solvent $\left(50-320{ }^{\circ} \mathrm{C}\right)$. The second stage was the degradation of the main chains of Pebax $\left(320-460{ }^{\circ} \mathrm{C}\right)$. It could be seen that the membranes doped with hZIF-L flakes had better thermal stability than the Pebax membrane. There was good interaction at the interface between the Pebax matrix and hZIF-L flakes, where the polymer chains exhibited partial chain rigidity, so a higher temperature was needed to break down the polymer chains, resulting in increasing the thermal stability.

\subsection{Performance of membrane separation}

3.3.1. Separation performance of pure gas. The pure gas $\left(\mathrm{CO}_{2}, \quad \mathrm{CH}_{4}\right)$ separation performance of Pebax/hZIF-L membranes was investigated. From Fig. 14(a), it could be seen that the Pebax/hZIF-L membrane exhibits higher separation performance compared to the pure Pebax and Pebax/ZIF-L membranes. Fig. 14(b) indicates that the permeability of the Pebax/hZIF-L membranes primarily increased with an increase in the loading of hZIF-L flakes, and it decreased when the content of hZIF-L flakes exceeded $7 \mathrm{wt} \%$. The increased $\mathrm{CO}_{2}$ permeability could be due to the presence of a large number of microporous structures on the hZIF-L flakes that promoted the diffusion of gas molecules. The selectivity of MMMs reached a maximum at a filler content of $5 \mathrm{wt} \%$, which was mainly attributed to the presence of $-\mathrm{COO}$ and $-\mathrm{OH}$ groups on the surface of the hZIF-L flakes, which showed high affinities for $\mathrm{CO}_{2}$ molecules.

The solubility and diffusion coefficients ( $S$ and $D$, respectively) of $\mathrm{CO}_{2}$ and $\mathrm{CH}_{4}$, and the selectivity for solubility and diffusion of the mixture, are shown in Table 1. Upon an increase in the filler content, the diffusion coefficient of $\mathrm{CO}_{2}$ molecules increased at first and then decreased, and the diffusion 


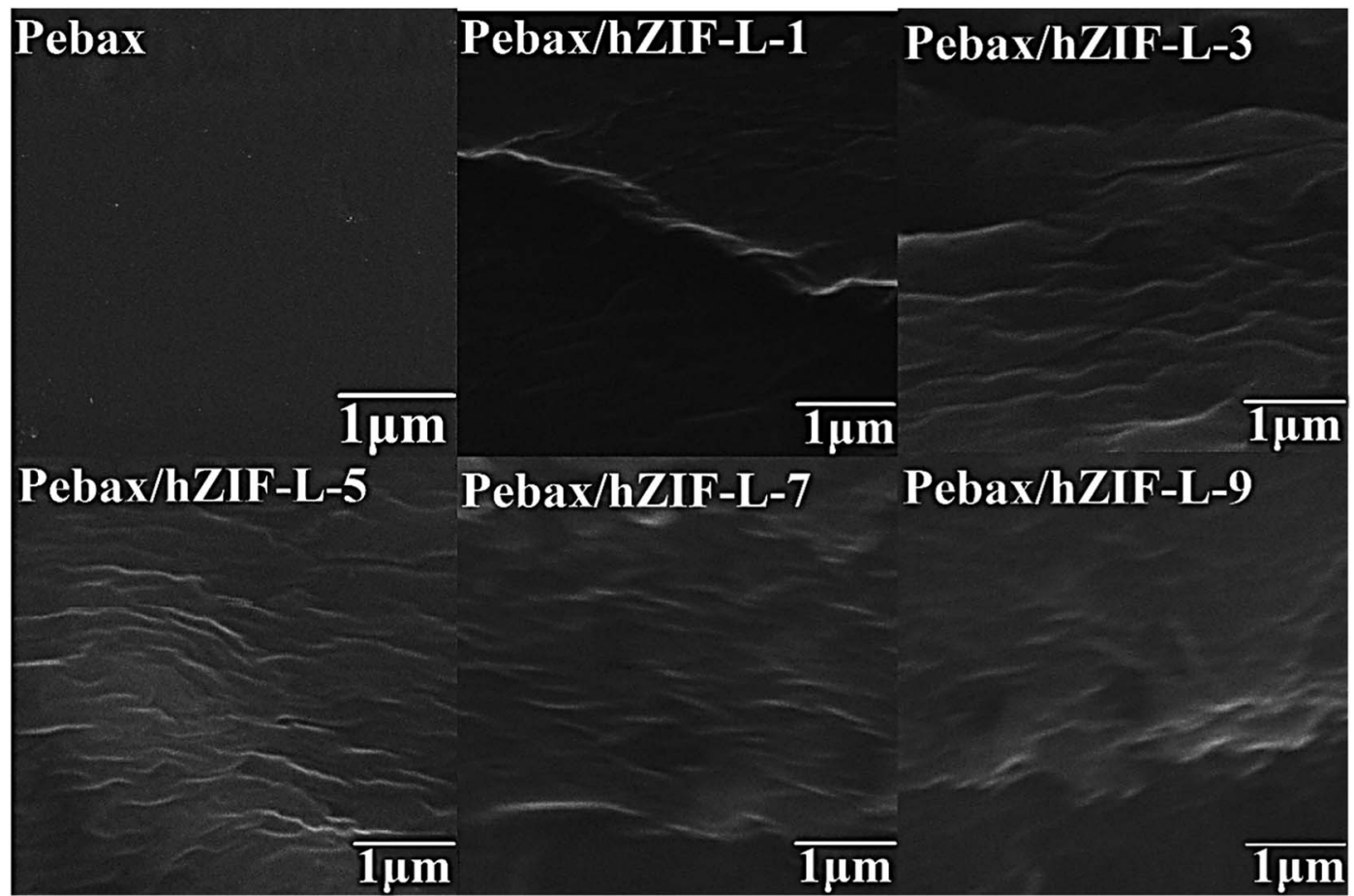

Fig. 9 SEM images of Pebax membranes with different contents of filler.

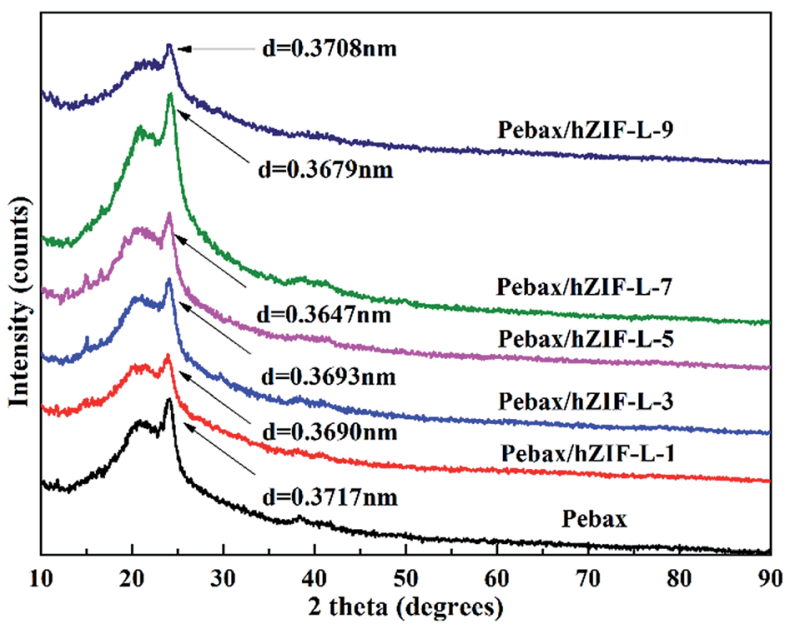

Fig. $10 \mathrm{XRD}$ patterns of membranes with different contents of filler.

coefficient of the $\mathrm{CH}_{4}$ molecule increased. This trend could be mainly due to the pore structure of the hZIF-L flakes, which promotes the diffusion of both molecules; but as the content increased, TA introduces more and more functional groups and the number of sites of action with $\mathrm{CO}_{2}$ increases, which limits the diffusion of $\mathrm{CO}_{2}$ molecules. Therefore, the diffusion selectivity of MMMs has an inverse relationship with the filler content. The solubility coefficient of $\mathrm{CO}_{2}$ molecules increased, and the solubility coefficient of $\mathrm{CH}_{4}$ molecules generally remains the same with an increase in the content of hZIF-L flakes. These results could be attributed to an increase in the content of pro- $\mathrm{CO}_{2}$ sites on the hZIF-L flakes, increasing the solubility selectivity of the MMMs. Comprehensively, the highest gas separation performance was achieved at $5 \mathrm{wt} \%$ loading of hZIF-L flakes in the polymer matrix with a $\mathrm{CO}_{2}$ permeability of 75.17 barrer and $\mathrm{CO}_{2} / \mathrm{CH}_{4}$ selectivity of 16.93 .

3.3.2. Separation performance of a gas mixture. Fig. 15 shows the effect of the content of ZIF-L and hZIF-L flakes of Pebax/ZIF-L and Pebax/hZIF-L membranes on the separation of a mixed gas. The Pebax/hZIF-L membranes show improved permeability for $\mathrm{CO}_{2}$ and selectivity for $\mathrm{CO}_{2} / \mathrm{CH}_{4}$ in relation to the pure Pebax and Pebax/ZIF-L membranes, where the separation performance originally increased with an increase in the loading of hZIF-L flakes and decreased when the content of

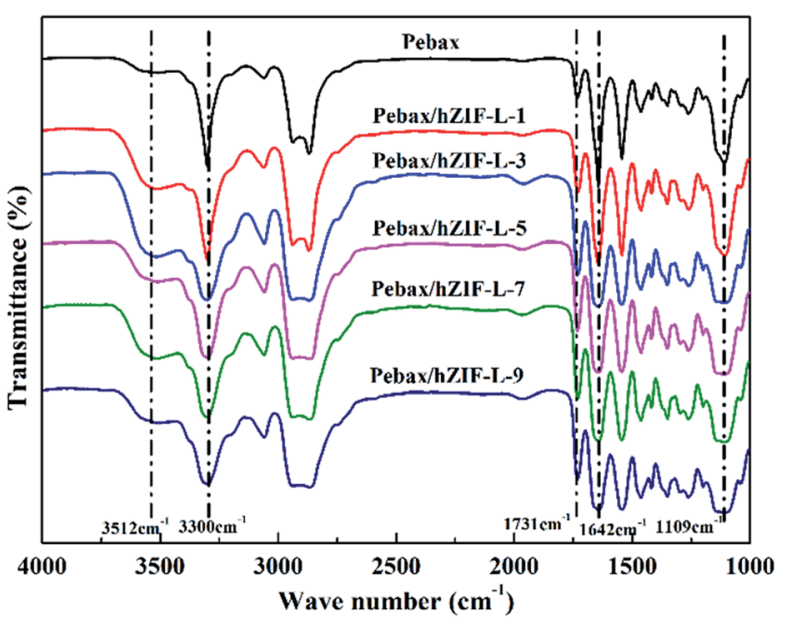

Fig. 11 FT-IR spectra of membranes with different contents of filler. 

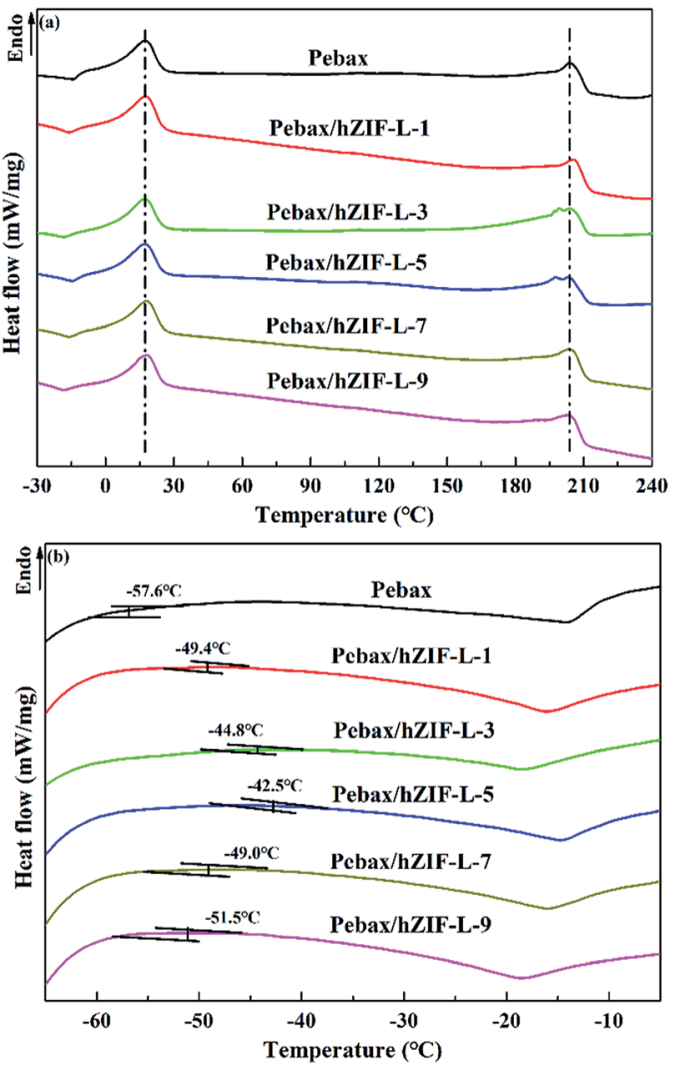

Fig. 12 DSC curves of membranes with different contents of filler: (a) high temperature region and (b) low temperature region.

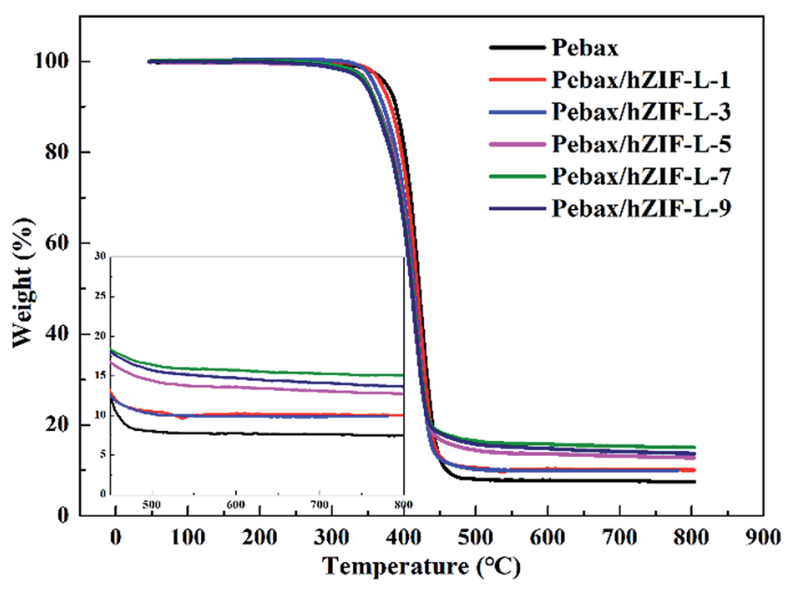

Fig. 13 TGA curves of membranes with different contents of filler.

hZIF-L flakes exceeded $5 \mathrm{wt} \%$. When hZIF-L flakes ( $5 \mathrm{wt} \%$ ) were doped into the Pebax matrix, the highest $\mathrm{CO}_{2}$ permeability was 502.44 barrer and the highest $\mathrm{CO}_{2} / \mathrm{CH}_{4}$ selectivity was 33.82 . There could be two major reasons for this: first, water is important in the separation of Pebax/hZIF-L membranes. Specifically, water could swell the Pebax polymer chain to increase its mobility, and thus the permeability of $\mathrm{CO}_{2}$ increased. Table 2 shows the results of the water contact angle measurements of the three membranes. A decrease in the water
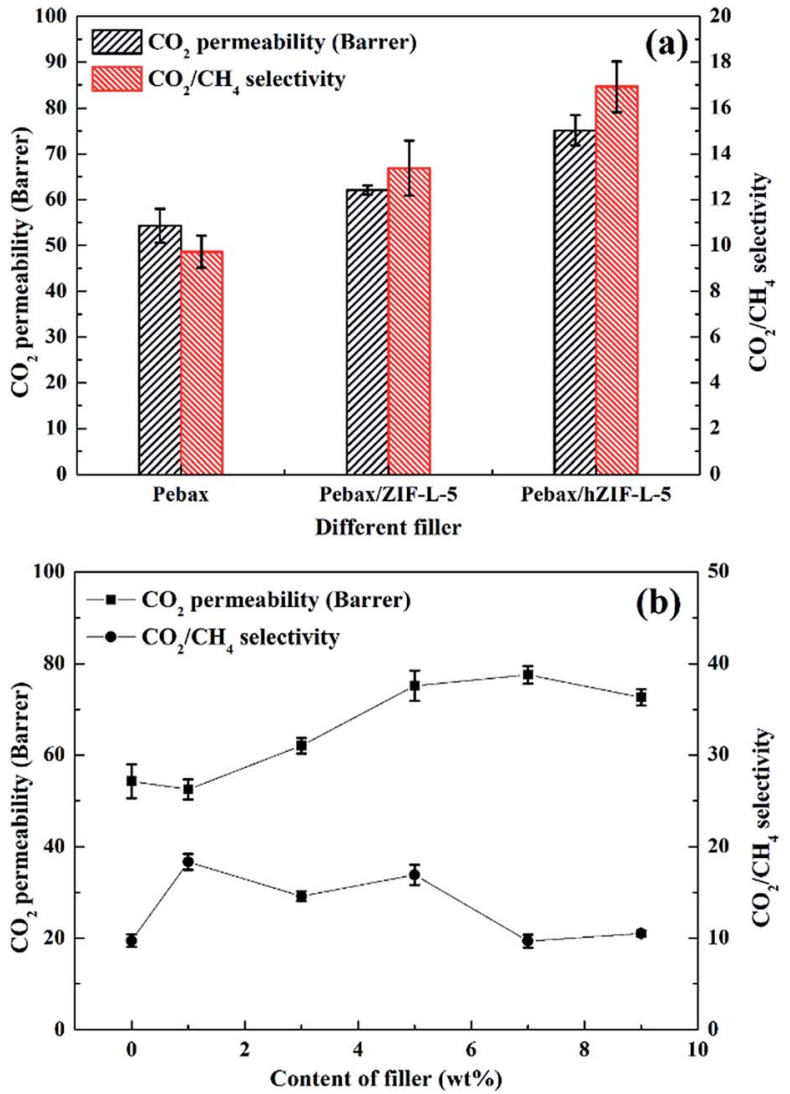

Fig. 14 Pure gas separation performance of Pebax/hZIF-L membranes: (a) different fillers and (b) different contents of filler.

Table 1 Gas diffusion and solubility coefficients of Pebax/hZIF-L membranes with different contents of filler ${ }^{a}$

\begin{tabular}{lccccll}
\hline Membrane & $D_{\mathrm{CO}_{2}}$ & $D_{\mathrm{CH}_{4}}$ & $S_{\mathrm{CO}_{2}}$ & $S_{\mathrm{CH}_{4}}$ & $D_{\mathrm{CO}_{2}} / D_{\mathrm{CH}_{4}}$ & $S_{\mathrm{CO}_{2}} / S_{\mathrm{CH}_{4}}$ \\
\hline Pebax & 3.95 & 5.08 & 1.37 & 0.11 & 0.78 & 12.45 \\
Pebax/hZIF-L-1 & 1.69 & 1.43 & 3.11 & 0.20 & 1.18 & 15.55 \\
Pebax/hZIF-L-3 & 2.09 & 2.25 & 2.98 & 0.19 & 0.93 & 15.68 \\
Pebax/hZIF-L-5 & 2.02 & 2.23 & 3.72 & 0.20 & 0.91 & 18.60 \\
Pebax/hZIF-L-7 & 1.96 & 3.53 & 3.97 & 0.23 & 0.56 & 17.26 \\
Pebax/hZIF-L-9 & 1.90 & 3.47 & 3.82 & 0.20 & 0.55 & 19.10
\end{tabular}

${ }^{a} D$ : diffusion coefficients, $\times 10^{-7} \mathrm{~cm}^{2} \mathrm{~s}^{-1} ; S$ : solubility coefficients, $\times 10^{-2} \mathrm{~cm}^{3}$ (STP) $\mathrm{cm}^{-3} \mathrm{cmHg}^{-1}$.

contact angles in the MMMs could be attributed to the $-\mathrm{COO}$ and -OH groups in the hZIF-L flakes that form hydrogen bonds with water. Secondly, the TA molecules endow the surface of the ZIF-L flakes with more hydrophilic functional groups such as $-\mathrm{COO}$ and $-\mathrm{OH}$, which could effectively prevent non-selective interfacial voids and agglomeration of filler in the Pebax matrix. The satisfactory interfacial compatibility and affinity with $\mathrm{CO}_{2}$ molecules increased the permeability and selectivity towards $\mathrm{CO}_{2}$. Based on these observations, the Pebax/hZIF-L-5 membrane was selected for subsequent conditional testing.

3.3.3. Effect of feed gas pressure on the gas separation performance. The permeability of the pure gases and the 


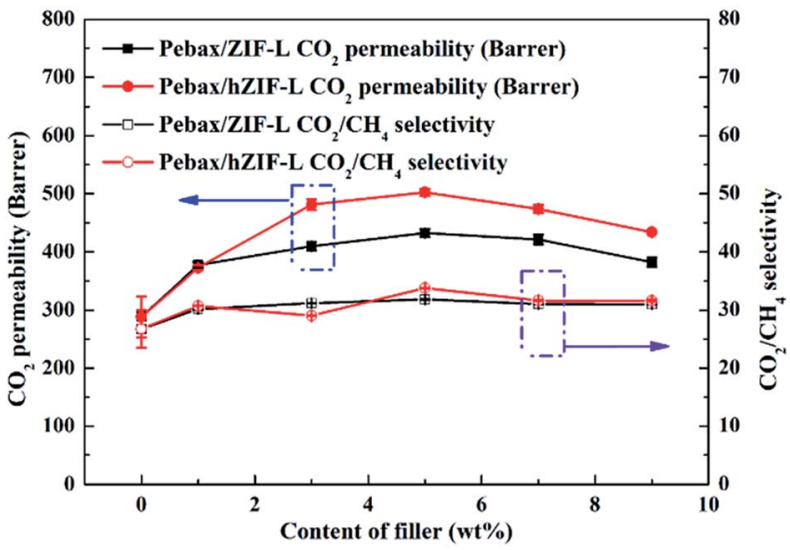

Fig. 15 Effect of the content of fillers on the mixed-gas separation performance of Pebax/hZIF-L membranes with different contents of filler.

Table 2 The water contact angles of the three membranes

\begin{tabular}{llll}
\hline Membrane & Pebax & Pebax/ZIF-L-5 & Pebax/hZIF-L-5 \\
\hline Contact angle $\left(^{\circ}\right)$ & 75.94 & 73.85 & 70.49
\end{tabular}

selectivity for the mixture are shown in Fig. 16. It could be seen from Fig. 16(a) that the permeability of the pure gases decreased with an increase in the pressure of feed gas. This result could be explained by the stacking of polymer chains, which causes all the membranes to become denser at high pressure, and limits the transport of gas molecules. Fig. 16(b) shows that the
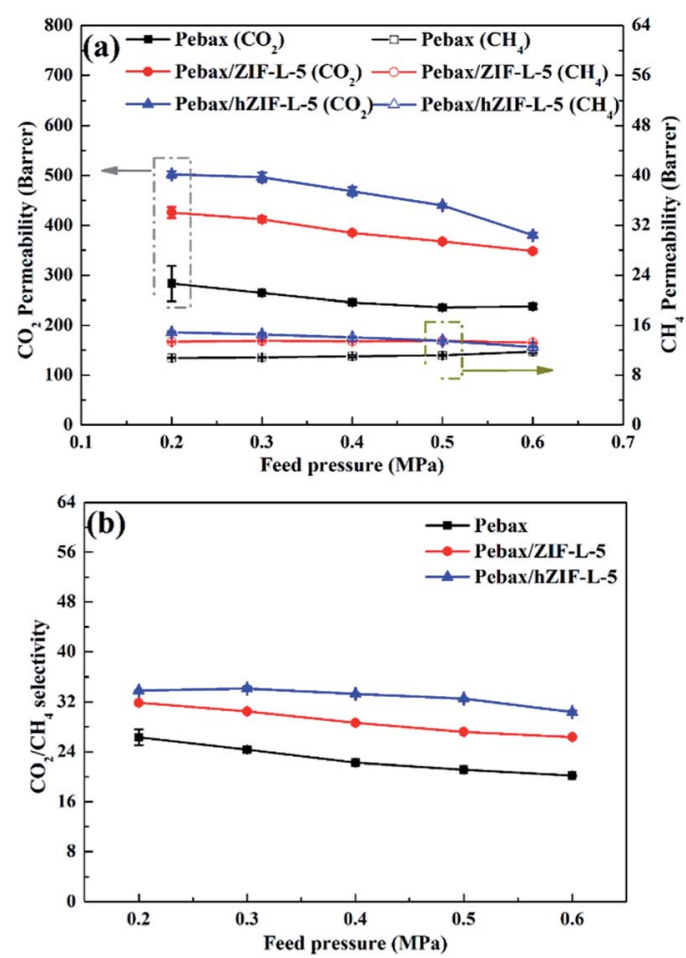

Fig. 16 Effect of feed gas pressure on the permeability of $\mathrm{CO}_{2}$ and $\mathrm{CH}_{4}$ (a) and selectivity (b).
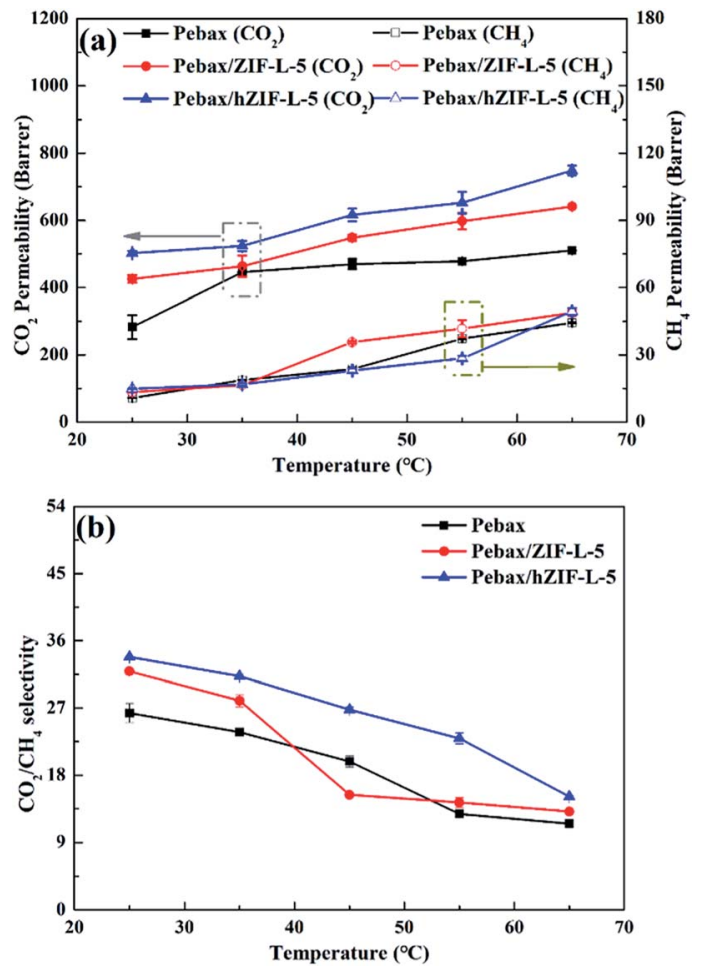

Fig. 17 Effect of operating temperature on the permeability of $\mathrm{CO}_{2}$ and $\mathrm{CH}_{4}$ (a) and selectivity (b).

selectivity decreased with an increase in the feed pressure. The number of $\mathrm{CO}_{2}$ molecules in contact with the polar groups of hZIF-L flakes and Pebax matrix decreased as all the membranes became denser at high feed pressure. In this way, the rate of decline in the permeability of $\mathrm{CO}_{2}$ molecules was greater than that of $\mathrm{CH}_{4}$ molecules, which led to a diminished selectivity. ${ }^{43}$ However, the Pebax/hZIF-L-5 membrane gave a better separation than the Pebax membrane, as the horizontal alignment of hZIF-L flakes in the Pebax matrix inhibited the mobility of the chains. ${ }^{44}$

3.3.4. Effect of operating temperature on the gasseparation performance. Fig. 17 shows the effect of temperature on the gas separation using the pure Pebax and Pebax/hZIFL-5 membranes. It could be seen from Fig. 17(a) that the permeability of $\mathrm{CO}_{2}$ and $\mathrm{CH}_{4}$ gradually increased with the operating temperature, caused by the faster gas diffusion and chain mobility of Pebax. It is shown in Fig. 17(b) that the selectivity of $\mathrm{CO}_{2} / \mathrm{CH}_{4}$ decreased at a higher operating temperature. This could be due to the decrease in the water content in the membranes at high temperature, which lowers the diffusion rate of $\mathrm{CO}_{2}$ molecules; and, in the same case, the diffusion rate of $\mathrm{CH}_{4}$ molecules was faster. However, the homogeneous dispersion of hZIF-L flakes limited the movement of Pebax chains, leading to a better gas separation using the Pebax/hZIFL-5 membrane as compared to the pure Pebax membrane.

\section{Conclusions}

In this investigation, ultra-thin ZIF-L flakes were successfully prepared by an ultrasonication method, and then the 
functionalized auxiliary etching of the ZIF-L surface was carried out using TA to form hZIF-L flakes. The TA etching of ZIF-L flakes not only enlarged the surface pores but also incorporated hydrophilic groups on the surface of the ZIF-L flakes. The hydrophilic groups had strong binding affinities for water and $\mathrm{CO}_{2}$ molecules, which improved the solubility, selectivity, and permeability of $\mathrm{CO}_{2}$. Also, the hZIF-L flakes showed good interfacial affinity with the polymer matrix. The mixed-gas test results at $0.2 \mathrm{MPa}$ and $25{ }^{\circ} \mathrm{C}$ gave a $\mathrm{CO}_{2}$ permeability of 502.44 barrer and a $\mathrm{CO}_{2} / \mathrm{CH}_{4}$ selectivity of 33.82 . Compared with the pure Pebax membrane, the $\mathrm{CO}_{2}$ permeability increased by $74.4 \%$, and the $\mathrm{CO}_{2} / \mathrm{CH}_{4}$ selectivity increased by $5.02 \%$ for Pebax/hZIF-L-5. The primary contribution of this work is that it provides a viable strategy for the application of hydrophobic ZIF-L materials in $\mathrm{CO}_{2} / \mathrm{CH}_{4}$ gas separation.

\section{Conflicts of interest}

There are no conflicts to declare.

\section{Acknowledgements}

The authors gratefully acknowledge the support from the National High Technology Research and Development Program of China (2012AA03A611) and the Program for Changjiang Scholars and Innovative Research Team in University (No. IRT15R46).

\section{References}

1 W. Zhu, Y. Qin, Z. Wang, J. Zhang, R. Guo and X. Li, J. Energy Chem., 2019, 31, 1-10.

2 N. Du, H. B. Park, M. M. Dal-Cin and M. D. Guiver, Energy Environ. Sci., 2012, 5, 7306-7322.

3 L. M. Robeson, J. Membr. Sci., 2008, 320, 390-400.

4 K. Yang, Y. Dai, W. Zheng, X. Ruan, H. Li and G. He, Sep. Purif. Technol., 2019, 214, 87-94.

5 X. Fu, X. Li, R. Guo, J. Zhang and X. Cao, High Perform. Polym., 2018, 30, 1064-1074.

6 D. Wu, C. Yi, Y. Wang, S. Qi and B. Yang, J. Membr. Sci., 2018, 551, 191-203.

7 P. Salehian, F. Wai and T. Chung, J. Membr. Sci., 2016, 518, 110-119.

8 N. Zhang, D. Peng, H. Wu, Y. Ren, L. Yang, X. Wu, Y. Wu, Z. Qu, Z. Jiang and X. Cao, J. Membr. Sci., 2018, 549, 670-679.

9 R. Xu, Z. Wang, M. Wang, Z. Qiao and J. Wang, J. Membr. Sci., 2019, 573, 455-464.

10 X. Li, L. Ma, H. Zhang, S. Wang, Z. Jiang, R. Guo, H. Wu, X. Cao, J. Yang and B. Wang, J. Membr. Sci., 2015, 479, 1-10.

11 L. Dong, M. Chen, J. Li, D. Shi, W. Dong, X. Li and Y. Bai, J. Membr. Sci., 2016, 520, 801-811.

12 J. Zhang, Q. Xin, X. Li, M. Yun, R. Xu, S. Wang, Y. Li, L. Lin, X. Ding, H. Ye and Y. Zhang, J. Membr. Sci., 2019, 570-571, 343-354.

13 J. E. Shin, S. K. Lee, Y. H. Cho and H. B. Park, J. Membr. Sci., 2019, 572, 300-308.
14 X. Li, Y. Cheng, H. Zhang, S. Wang, Z. Jiang, R. Guo and H. Wu, ACS Appl. Mater. Interfaces, 2015, 7, 5528-5537.

15 S. Quan, S. Li, Y. Xiao and L. Shao, Int. J. Greenhouse Gas Control, 2017, 56, 22-29.

16 J. Hou, X. Li, R. Guo, Z. Wang and J. Zhang, Nanotechnology, 2018, 29, 125706.

17 B. P. Biswal, H. D. Chaudhari, R. Banerjee and U. K. Kharul, Chem.-Eur. J., 2016, 22, 4695-4699.

18 Z. Kang, Y. Peng, Y. Qian, D. Yuan, M. A. Addicoat, T. Heine, Z. Hu, L. Tee, Z. Guo and D. Zhao, Chem. Mater., 2016, 28, 1277-1285.

19 Y. Liu, C. Chen, G. Lin, C. Chen, K. C. Wu, C. Lin and K. Tung, J. Membr. Sci., 2019, 582, 358-366.

20 M. Zhao, Q. Lu, Q. Ma and H. Zhang, Small Methods, 2017, 1, 1600030.

21 T. Rodenas, I. Luz, G. Prieto, B. Seoane, H. Miro, A. Corma, F. Kapteijn, F. X. L. Xamena and J. Gascon, Nat. Mater., 2015, 14, 48-55.

22 Y. Cheng, X. Wang, C. Jia, Y. Wang, L. Zhai, Q. Wang and D. Zhao, J. Membr. Sci., 2017, 539, 213-223.

23 G. Huang, A. P. Isfahani, A. Muchtar, K. Sakurai, B. B. Shrestha, D. Qin, D. Yamaguchi, E. Sivaniah and B. Ghalei, J. Membr. Sci., 2018, 565, 370-379.

24 D. Zhao, J. Ren, Y. Qiu, H. Li, K. Hua, X. Li and M. Deng, J. Appl. Polym. Sci., 2015, 132, 42624.

25 R. Chen, J. Yao, Q. Gu, S. Smeets, C. Baerlocher, H. Gu, D. Zhu, W. Morris, O. M. Yaghi and H. Wang, Chem. Commun., 2013, 49, 9500.

26 B. Motevalli, H. Wang and J. Z. Liu, J. Phys. Chem. C, 2015, 119, 16762-16768.

27 B. Motevalli, N. Taherifar, H. Wang and J. Z. Liu, J. Phys. Chem. C, 2017, 121, 2221-2227.

28 S. Kim, E. Shamsaei, X. Lin, Y. Hu, G. P. Simon, J. G. Seong, J. S. Kim, W. H. Lee, Y. Moo-Lee and H. Wang, J. Membr. Sci., 2018, 549, 260-266.

29 M. Hu, Y. Ju, K. Liang, T. Suma, J. Cui and F. Caruso, Adv. Funct. Mater., 2016, 26, 5827-5834.

30 C. Lin, M. Zhou, W. Hung, B. Zhu, K. Lee, L. Zhu and L. Fang, Sep. Purif. Technol., 2018, 207, 435-442.

31 H. Sun, B. Tang and P. Wu, J. Membr. Sci., 2018, 551, 283293.

32 K. Duan, J. Wang, Y. Zhang and J. Liu, J. Membr. Sci., 2019, 572, 588-595.

33 W. Zheng, R. Ding, K. Yang, Y. Dai, X. Yan and G. He, Sep. Purif. Technol., 2019, 214, 111-119.

34 Z. Wang, J. Hou, R. Guo and X. Li, J. Chin. Chem. Soc., 2018, 65, 1347-1355.

35 H. Zhang, R. Guo, J. Zhang and X. Li, ACS Appl. Mater. Interfaces, 2018, 10, 43031-43039.

36 H. Zhang, R. Guo, J. Hou, Z. Wei and X. Li, ACS Appl. Mater. Interfaces, 2016, 8, 29044-29051.

37 Z. Low, A. Razmjou, K. Wang, S. Gray, M. Duke and H. Wang, J. Membr. Sci., 2017, 460, 9-17.

38 G. Liu, Z. Jiang, K. Cao, S. Nair, X. Cheng, J. Zhao, H. Gomaa, H. Wu and F. Pan, J. Membr. Sci., 2016, 523, 185-196.

39 L. Valencia and H. N. Abdelhamid, Carbohydr. Polym., 2019, 213, 338-345. 
40 H. N. Abdelhamid and X. Zou, Green Chem., 2018, 20, 10741084.

41 Y. Li, H. Guan, T. Chung and S. Kulprathipanja, J. Membr. Sci., 2006, 275, 17-28.

42 Y. Cheng, Y. Ying, L. Zhai, G. Liu, J. Dong, Y. Wang, M. P. Christopher, S. Long, Y. Wang and D. Zhao, J. Membr. Sci., 2019, 573, 97-106.
43 R. Vaidhyanathan, S. S. Iremonger, G. K. H. Shimizu, P. G. Boyd, S. Alavi and T. K. Woo, Science, 2010, 330, 650653.

44 Q. Xin, T. Liu, Z. Li, S. Wang, Y. Li, Z. Li, J. Ouyang, Z. Jiang and H. Wu, J. Membr. Sci., 2015, 488, 67-78. 